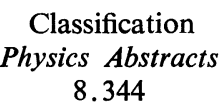

\title{
ÉTUdE DE LA DIFFUSION DE L'ARGENT DANS LES CRISTAUX DE CdS PAR LA MÉTHODE CAPACITIVE
}

\author{
J.-P. SORBIER et N. SANGUINETTI \\ Laboratoire de Photoélectricité, Université de Provence, \\ 13397 Marseille Cedex 4, France
}

(Reçu le 29 avril 1974, révisé le 20 juin 1974)

\begin{abstract}
Résumé. - La méthode capacitive permet de suivre l'évolution du profil de la charge d'espace du contact métal-semiconducteur occasionnée par la diffusion. Le but de ce travail est de tester cette méthode en étudiant la diffusion de l'argent dans le $\mathrm{CdS}$ entre $100^{\circ} \mathrm{C}$ et $300^{\circ} \mathrm{C}$ avec des temps de recuits inférieurs à $20 \mathrm{~h}$.

Abstract. - The capacitance method allows us to follow the evolution of the space-charge profile of a metal semiconductor contact due to diffusion. The aim of our work is to test this method by studying the diffusion of silver in CdS crystals between $100^{\circ} \mathrm{C}$ and $300^{\circ} \mathrm{C}$ with firing times below $20 \mathrm{~h}$.
\end{abstract}

Introduction. - L'étude des photopiles au CdS$\mathrm{Cu}_{2} \mathrm{~S}$ en couches minces a montré toute l'importance des phénomènes de diffusion matérielle du cuivre [1].

Ces phénomènes de diffusion dans les couches minces déterminent aussi bien les performances immédiates de ces convertisseurs que leur fiabilité. C'est pourquoi leur étude détaillée s'est imposée.

Pour éviter de se heurter aux difficultés qu'introduisent les couches minces polycristallines du fait de leurs imperfections (joints de grains, dislocations, ...), nous avons commencé cette étude sur des monocristaux de CdS.

Pour ne pas s'écarter des conditions de préparation des "photopiles, nous devons opérer à des températures relativement basses $\left(100-300^{\circ} \mathrm{C}\right)$, domaines dans lequel l'étude de la diffusion par la méthode conventionnelle des traceurs radioactifs n'est pas possible, car elle nécessite une activité spécifique minimum conditionnée par la sensibilité de l'échelle de comptage et par la qualité du parallélisme des abrasions pratiquées. Pour atténuer ces inconvénients, nous devons avoir des profondeurs de pénétration supérieures à $10 \mu$, profondeurs qui sont obtenues avec des températures supérieures à $250^{\circ} \mathrm{C}$ d'une part et par des temps de recuit supérieurs à $24 \mathrm{~h}$ d'autre part. Or, le rendement maximum d'une photopile est obtenu pour des températures variant autour de $200^{\circ} \mathrm{C}$ et pour des temps de recuit de quelques heures, c'est-à-dire que nous devons nous intéresser à une zone comprise entre 0 et $5 \mu$ d'épaisseur. Ainsi, pour mesurer le profil de diffusion, nous avons utilisé une méthode de mesure capacitive non destructive de l'échantillon et plus sensible que la méthode des traceurs radioactifs.

Cette méthode permet de suivre l'évolution du profil de la charge d'espace du contact métal-semiconducteur occasionnée par la diffusion.

Le but de ce travail est de tester cette méthode en étudiant la diffusion de l'argent dans le CdS entre $100^{\circ} \mathrm{C}$ et $300^{\circ} \mathrm{C}$ avec des temps de recuit inférieurs à $20 \mathrm{~h}$.

Dans ce qui suit, nous exposerons le principe de la méthode capacitive, la technique expérimentale et les résultats des mesures, ainsi que les diverses conclusions auxquelles nous sommes actuellement parvenus.

1. Théorie du contact métal-semiconducteur. 1.1 Charge D'espaCe eT BARRIÈre DE POTENTIEL. La hauteur de barrière pour le passage des électrons du métal vers le semiconducteur est :

$$
\varphi_{\mathrm{B}}=\varphi_{\mathrm{m}}-\chi,
$$

$\chi$ étant l'affinité électronique (Fig. 1a) tandis que la barrière pour le flux inverse est :

$$
V_{\mathrm{D}}=\varphi_{\mathrm{m}}-\varphi_{\mathrm{s}},
$$

où $V_{\mathrm{D}}$ est le potentiel de diffusion caractéristique du contact.

1.2 DENSITÉ DE DONNEURS CONSTANTE. - Si la concentration d'électrons libres est négligeable devant 


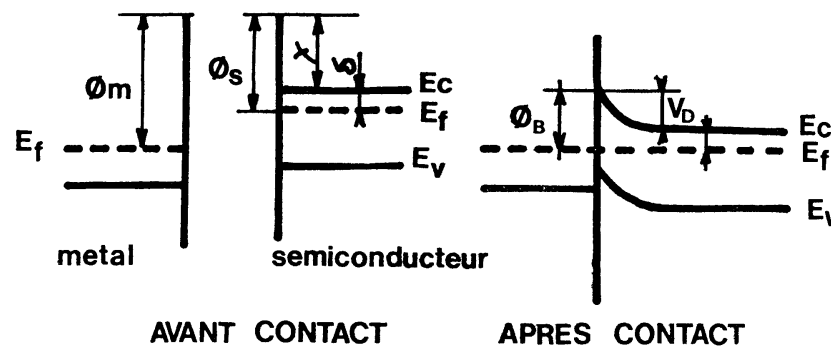

FIG. 1a. - Schéma de bandes du contact métal-semiconducteur.

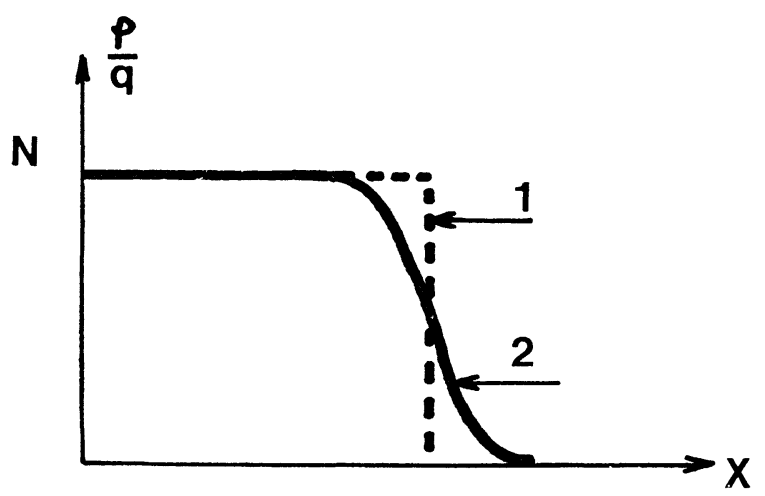

Fig. 1b. - Courbe 1 : Variation de la densité de charge dans la zone de charge d'espace. Courbe 2: Approximation abrupte de Schottky.

$N_{\mathrm{D}}$, l'approximation de Schottky (Fig. $1 b$ ) est valable. $\mathrm{La}$ formule

$$
\frac{1}{C^{2}}=\frac{2\left(V_{\mathrm{D}}-V\right)}{q \varepsilon N_{\mathrm{D}} S^{2}}
$$

nous montre que la variation de $1 / C^{2}=f(V)$ est linéaire.

La pente de la droite nous permet de calculer $N_{\mathrm{D}}$ dons $\delta$ intervalle que sépare le niveau de Fermi au bas de la bande de conduction ainsi que $V_{\mathrm{D}}$ obtenu par extrapolation de la courbe $1 / C^{2}=f(V)=0$.

1.3 Densité De DonNeurs variable. - Supposons que la densité d'électrons libres soit négligeable à l'intérieur de la zone de charge d'espace et que la densité de donneurs suive une loi $N(x)$ quelconque. Dans ce cas, l'équation de Poisson s'écrit :

$$
\frac{\mathrm{d}^{2} V(x)}{\mathrm{d} x^{2}}=-\frac{q}{\varepsilon} N(x)
$$

pour la tension de polarisation $V$ appliquée, le bord de la zone de la charge d'espace est à $x=l$.

Le champ électrique à l'intérieur de cette zone vaut :

$$
E=-\frac{\mathrm{d} V}{\mathrm{~d} x}=\frac{q}{\varepsilon} \int_{l}^{x} N(x) \mathrm{d} x
$$

et la charge totale est :

$$
Q=q S \int_{0}^{l} N(x) \mathrm{d} x .
$$

E La tension aux bornes du contact est :

$$
V_{\mathrm{D}}-V=\frac{q}{\varepsilon} \int_{0}^{l}\left[\int_{x}^{l} N(x) \mathrm{d} x\right] \mathrm{d} x .
$$

Une variation de la tension de polarisation de $\mathrm{d} V$ se traduit par une variation de $\mathrm{d} l$ de la largeur de la zone de charge et par conséquent une variation $\mathrm{d} Q$ de la charge d'espace. Les éq. (1.4) et (1.5) donnent :

$$
\begin{aligned}
\mathrm{d} Q & =q S N(l) \mathrm{d} l \\
\mathrm{~d} V & =\frac{q}{\varepsilon} l N(l) \mathrm{d} l .
\end{aligned}
$$

On en déduit la capacité du contact :

$$
C=\left|\frac{\mathrm{d} Q}{\mathrm{~d} V}\right|=\frac{\varepsilon S}{l} .
$$

Les éq. (1.6) et (1.7) conduisent à :

$$
\frac{\mathrm{d}\left(1 / C^{2}\right)}{\mathrm{d} V}=-\frac{2}{q \varepsilon S^{2} N(l)} .
$$

Cette équation relie la densité de donneurs à la pente en chaque point du graphe

$$
\frac{1}{C^{2}}=f(V)
$$

point dont l'abscisse $V$ est la tension pour laquelle la zone de charge d'espace a l'épaisseur $l$.

L'éq. (1.7) montre que la capacité est proportionnelle à l'inverse de la distance $l$. Le tracé du graphe

$$
\frac{\mathrm{d} V}{\mathrm{~d}\left(1 / C^{2}\right)}=f\left(\frac{1}{C}\right)
$$

donnera l'allure du profil du dopage du semiconducteur au niveau du contact.

\subsection{CAPACITÉ DU CONTACT MÉTAL-SEMICONDUCTEUR} APRÈS DIFFUSION. - 1.4.1 Equation de Fick et ses solutions. - Un traitement thermique du contact fait diffuser des atomes du métal vers le semiconducteur. La concentration des atomes diffusés dans le cristal est décrite théoriquement par l'équation de Fick [2]

$$
\frac{\partial N}{\partial t}=-D \frac{\partial^{2} N}{\partial x^{2}}
$$

laquelle avec les conditions initiales

$$
\begin{array}{lll}
N=N_{0} ; & x=0, & t=0 \\
N=0 ; & x>0, & t=0
\end{array}
$$


admet pour solution :

$$
N(x, t)=N_{0} \operatorname{erfc}\left(\frac{x}{2 \sqrt{D t}}\right) .
$$

Cette équation décrit la distribution de la matière diffusante dans un matériau semi-infini avec un réservoir de matière diffusante $N_{0}$ constant à la surface.

Si les conditions initiales sont telles que l'épaisseur du dépôt est très petit devant $\sqrt{D t}$, la solution analytique de l'équation de Fick est particulièrement simple ; les courbes de concentration $N(x, t)$ sont des gaussiennes.

On écrit :

$$
N(x, t)=\frac{N_{\mathrm{s}}}{\sqrt{\pi D t}} \exp \left(-\frac{x^{2}}{4 D t}\right) .
$$

Cette solution satisfait les conditions initiales suivantes :

$$
\begin{gathered}
N=N_{\mathrm{s}} ; \quad x=0, \quad t=0 \\
\int_{0}^{+\infty} N(x, t) \mathrm{d} x=N_{\mathrm{s}}, \quad t>0 .
\end{gathered}
$$

Ces conditions expriment le fait que toute la matière diffusante est rassemblée dans le plan $x=0$ au temps $t=0$.

\subsubsection{Charge d'espace et répartition du potentiel} après diffusion. - Si nous avons compensation des donneurs par les atomes métalliques diffusés, l'équation de Poisson qui décrit le potentiel électrostatique du contact est alors

$$
\frac{\mathrm{d}^{2} V(x)}{\mathrm{d} x^{2}}=\frac{q}{\varepsilon}\left[N_{\mathrm{D}}-N(x, t)\right] .
$$

On peut intégrer cette équation avec les conditions initiales et aux limites du contact métal-semiconducteur. Si on utilise l'éq. (1.9), on obtient :

$$
\begin{aligned}
V_{\mathrm{D}}-V & =\frac{q N_{0}}{\varepsilon}\left[\frac{N_{\mathrm{D}}}{2 N_{0}} l^{2}+\right. \\
& +\frac{l \sqrt{D t}}{\pi} \exp \left(-\frac{l^{2}}{4 D t}\right) \\
& \left.-l^{2} \operatorname{erfc}\left(\frac{l}{2 \sqrt{D t}}\right)-D t \operatorname{erf}\left(\frac{l}{2 \sqrt{D t}}\right)\right] .
\end{aligned}
$$

Par contre, en utilisant l'éq. (1.10), la solution est :

$$
\begin{aligned}
V_{\mathrm{D}}-V=\frac{q}{\varepsilon}\left[N_{\mathrm{D}} l^{2}\right. & +\frac{4 D t}{\pi} N_{\mathrm{s}} \times \\
& \left.\times\left\{\exp \left(-\frac{l^{2}}{4 D t}\right)-1\right\}\right] .
\end{aligned}
$$

Lorsqu'on substitue $l$ par $\varepsilon S / C$ dans les éq. (1.11) et (1.12), celles-ci décrivent ainsi la variation de la capacité après diffusion en fonction de la tension appliquée, car nous avons démontré dans le $\S 1.3$, que pour une densité variạble de donneurs, la capacité est toujours donnée par l'éq. (1.7).

2. Préparation et caractéristiques des cristaux utilisés. - Les monocristaux de CdS utilisés ont été fournis en forme de barreau par la firme Eagle-Picher (Etats-Unis).

Les barreaux de départ ont été découpés à la scie diamantée en plaquettes de $2 \mathrm{~mm}$ d'épaisseur perpendiculairement à l'axe $C$. Ces plaquettes sont ensuite polies sur papier abrasif, puis sur tissu avec de la pâte diamantée (finesse $7 \mu$ ). Sur ces échantillons, par des mesures d'effet Hall, nous avons déterminé une densité moyenne de donneurs de $1,23 \times 10^{16} \mathrm{~cm}^{-3}$ et une mobilité électronique moyenne de

$$
220 \mathrm{~cm}^{2} \cdot \mathrm{V}^{-1} \mathrm{~s}^{-1} \text {. }
$$

Afin d'éliminer l'écrouissage des surfaces laissé par les papiers abrasifs, nous avons soumis les échantillons à un traitement chimique consistant à les nettoyer avec de l'acide chlorhydrique.

Ce traitement nous a permis de mettre en évidence le caractère polaire des échantillons (voir photos et Fig. 2). Sur la face brillante du cristal, les figures

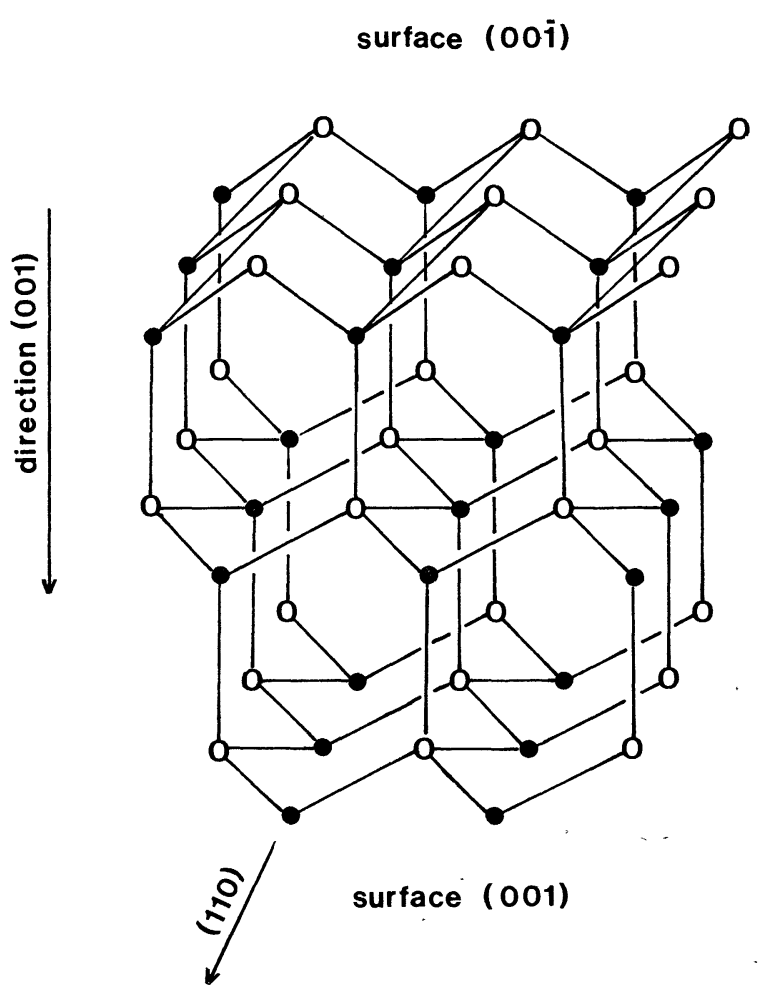

FIG. 2. - Structure polaire de la wurtzite. La face (001) repérée par des points est supposée être constituée par des atomes de cadmium et la face (001) par des atomes de soufre. 


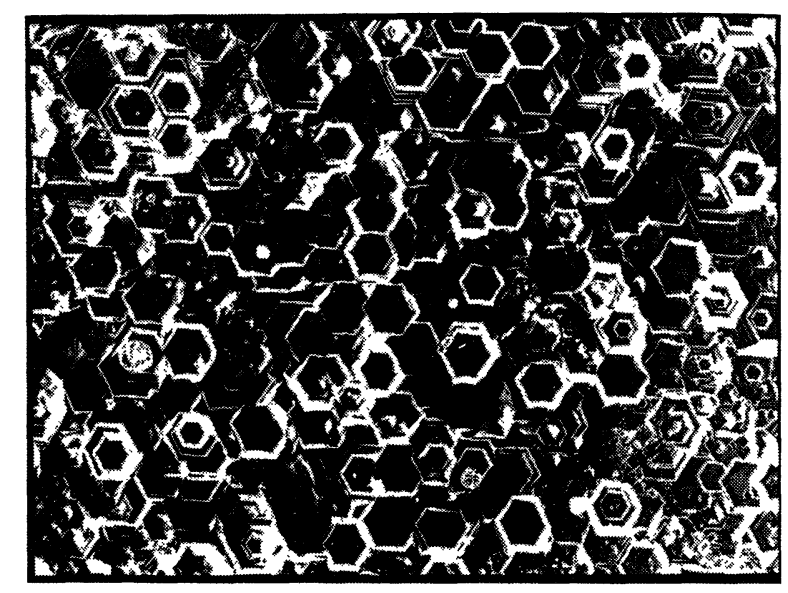

Рното 1. - Face cadmium. Les figures d'attaque hexagonales sont très nettes.

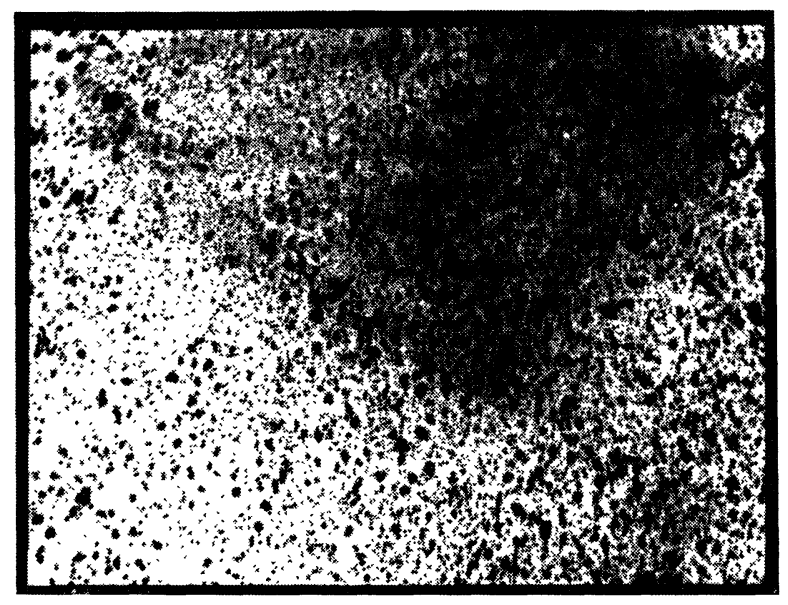

Рното 2. - Face soufre.

d'attaque présentent un caractère hexagonal très net et sur la face mate aucune figure de corrosion bien définie n'apparaît.

Suivant Haas, Fox et Katz [3], qui citent le travail de Wolff [4], la face brillante serait formée uniquement par des atomes de $\mathrm{Cd}$, et la face mate par des atomes de soufre.

Nos cristaux ont été nettoyés en les trempant dans de l'acide chlorhydrique pur pendant $2 \mathrm{~min}$.

2.1 RÉAlisATION DES DIODES. - Le métal utilisé pour le contact ohmique est de l'indium. La soudure In-CdS est réalisée sous vide au four $\mathrm{HF}$ par fusion d'une pastille d'indium plaquée sur la tranche des monocristaux de CdS (Fig. 3).

Après avoir réalisé le contact ohmique, nous avons effectué toutes les opérations de polissage et nettoyage précédemment citées.

Tous les dépôts métalliques d'argent ont été faits sur la face cadmium. En effet, une étude de l'influence des faces cadmium ou soufre sur les caractéristiques

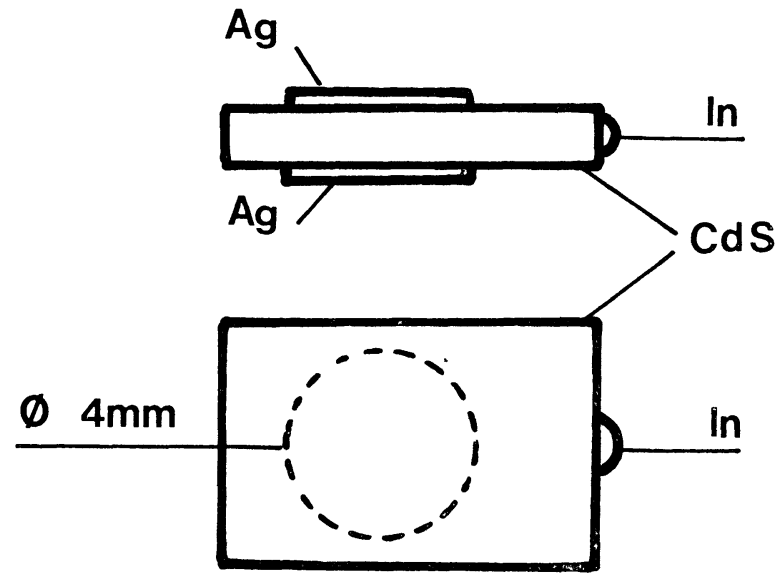

Fig. 3. - Schéma des diodes réalisées.

$1 / C^{2}=f(V)$ nous a montré que seule la face cadmium nous permet d'obtenir une barrière de Schottky. Ceci a été vérifié aussi bien pour l'argent que pour l'or. Comme le montre la figure 4, et contrairement à ce que trouve Sullivan [5], nous n'avons jamais mesuré deux capacités identiques sur les deux faces.

Nous n'effectuons pas de dégazage sous vide pour éviter une dissociation superficielle du CdS.

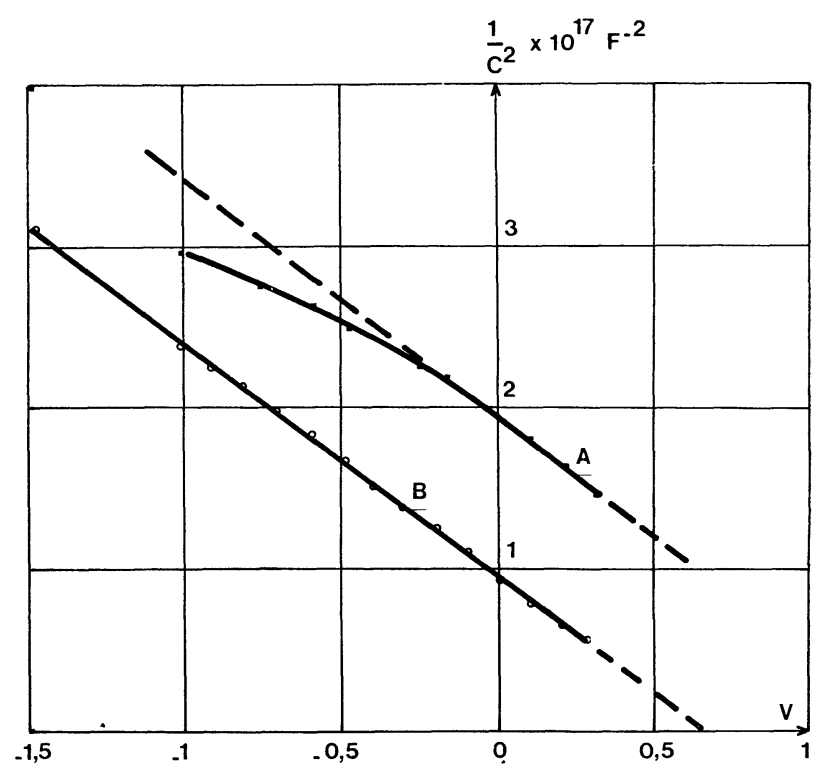

Fig. 4. - Variation de $1 / C^{2}$ en fonction de la tension appliquée. Diode AuCdSIn avant recuit. Courbe A face soufre ; courbe B face cadmium.

2.2 Montage ÉleCtRIQUE ET MeSURES RÉAliséES. Les diodes ainsi réalisées sont placées dans un cryostat pouvant fonctionner de 77 à $800 \mathrm{~K}$. Les recuits successifs sont faits sous une pression de $10^{-6}$ torr.

Sur chaque échantillon, nous avons mesuré la capacité du contact en fonction de la tension de polarisation, avant et après chaque recuit, sous obscurité et à température ambiante. 
Pour respecter les mêmes conditions initiales, nous avons dû fixer la température et faire varier le temps de recuit, par exemple : $5 \mathrm{~min} ; 1 \mathrm{~h}$; etc..., ce qui nous a permis de suivre l'évolution de nos diodes à une température déterminée.

Les mesures capacitives sont faites à l'aide d'un pont Général-Radio, type $1625 \mathrm{~A}$, qui donne la capacité et le facteur de perte, avec une précision absolue de $10^{-4}$. Les capacités parasites sont de l'ordre de quelques picofarads. Le signal alternatif d'attaque est inférieur à $10 \mathrm{mV}$ et sa fréquence de $100 \mathrm{kHz}$.

3. Résultats expérimentaux. - 3.1 INTRODUCTION. - Avant de procéder à un examen détaillé des résultats obtenus, quelques remarques sur la méthode capacitive pour l'étude de la diffusion doivent être rappelées.

La méthode capacitive permet de suivre l'évolution de la charge d'espace après diffusion. Il est cependant nécessaire d'avoir avant toute diffusion les conditions initiales imposées par le modèle de Schottky, c'est-à-dire une concentration de donneurs constante dans tout l'échantillon, ce qui entraîne une variation linéaire de $1 / C^{2}$ en fonction de $V$ (voir formule (1.1)).

Si on observe une déviation de cette linéarité avant recuit, nous ne pouvons pas établir les caractéristiques initiales de l'échantillon et il est impossible d'utiliser celui-ci pour étudier la diffusion.

3.2 RÉSULTATS OBTENUS POUR LES CONTACTS D'ARGENT. - 3.2.1 Avant diffusion. - Avant recuit, les diodes présentent une variation linéaire de $1 / C^{2}=f(V)$ analogue à celle de la figure 5 . Les pentes des droites donnent une densité moyenne de donneurs sur les échantillons étudiés de

$$
N_{\mathbf{D}}=7,5 \pm 0,25 \times 10^{15} \mathrm{~cm}^{-3}
$$

et des valeurs $V_{\mathrm{I}}$ interceptées sur l'axe des $V$, on déduit un potentiel de diffusion :

$$
V_{\mathbf{D}}=0,44 \pm 0,02 \mathrm{~V} \text {. }
$$

La profondeur du niveau de Fermi calculée à partir de $N_{\mathrm{D}}$ est de $\delta=0,142 \pm 0,002 \mathrm{eV}$ sous la bande de conduction, ce qui conduit à une hauteur de barrière moyenne

$$
\varphi_{\mathrm{B}}=0,58 \mathrm{eV} \pm 0,02 \mathrm{eV} .
$$

Nous avons calculé $\varphi_{\mathrm{B}}$ en utilisant la caractéristique $I=f(V)$ par application de la théorie de l'émission thermoélectronique de Bethe [6].

Nous obtenons une hauteur moyenne $\varphi_{\mathrm{B}}=0,52 \mathrm{eV}$, soit un tout petit peu moins que le $\varphi_{\mathbf{B}}$ obtenu par la caractéristique $1 / C^{2}=f(V)$. Cet écart est dû à la force image qui abaisse la hauteur de barrière d'une grandeur $\Delta \varphi=0,04 \mathrm{eV}$ pour un champ électrique appliqué de $10^{4} \mathrm{~V} \mathrm{~cm}^{-1}$.

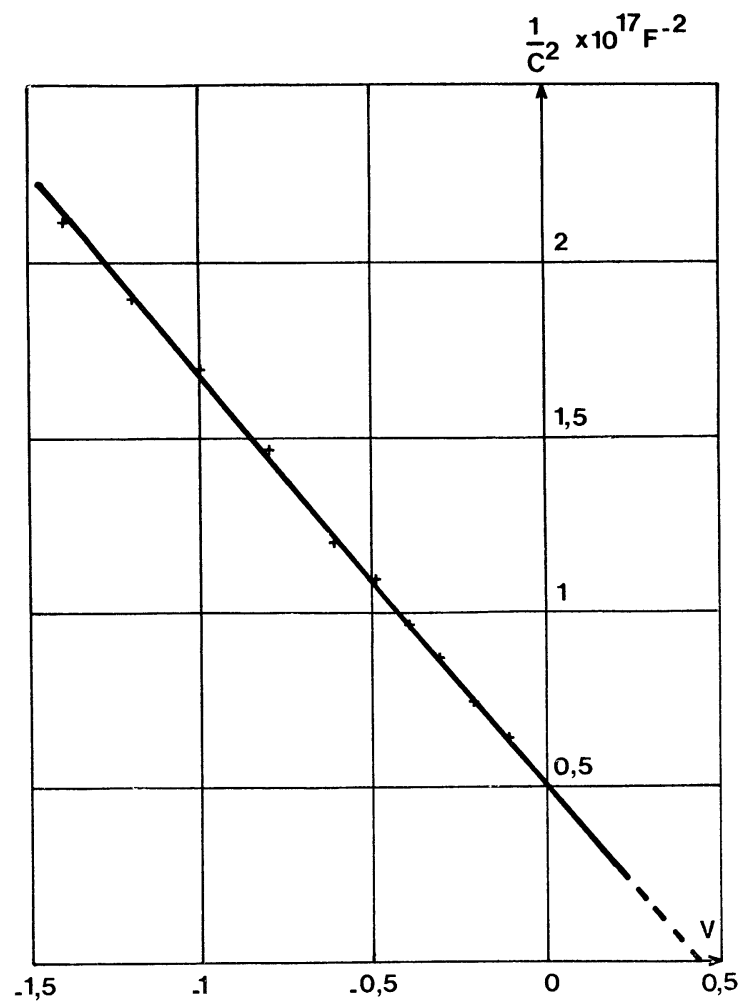

FIG. 5. - Variation de $1 / C^{2}$ en fonction de la tension appliquée. Diode AgCdSIn avant recuit.

3.2.2 Après diffusion. - Les formes générales des variations de $1 / C^{2}=f(V)$ des contacts après la diffusion de l'argent à différentes températures sont données par la figure 6.

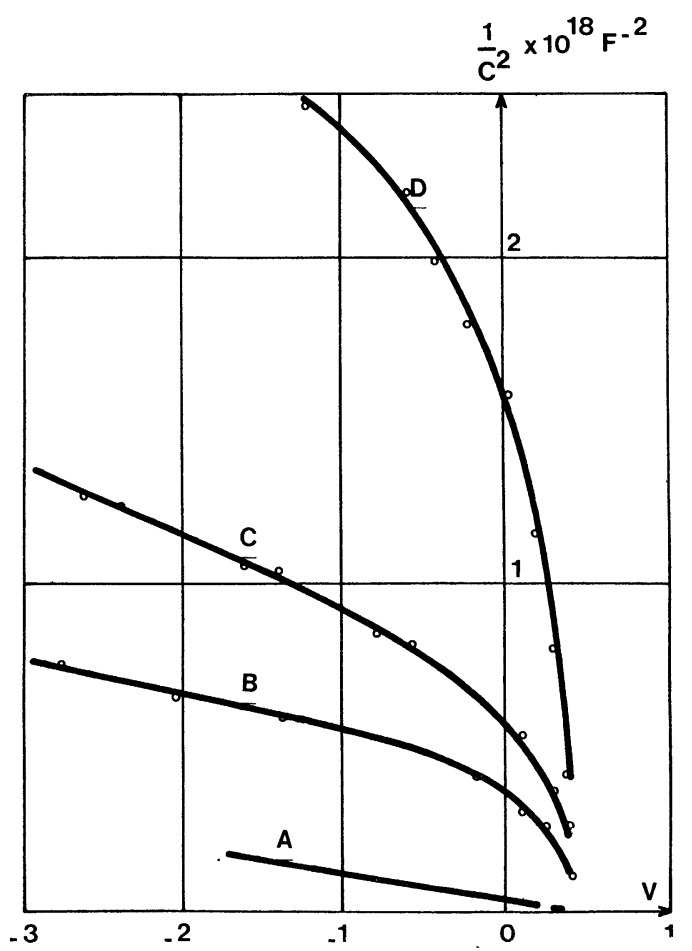

Fig. 6. - Variation de $1 / C^{2}$ en fonction de la tension appliquée. Diode AgCdSIn. Courbe A avant recuit. Courbe B recuit $100^{\circ} \mathrm{C}$ 18 h. Courbe $C$ recuit $200^{\circ} \mathrm{C} 10$ h. Courbe D recuit $300^{\circ} \mathrm{C} 6 \mathrm{~h}$. 
La relation (1.8) nous permet de tracer à partir des courbes précédentes les courbes des concentrations des donneurs au niveau du contact. Ceci est représenté sur la figure 7 pour les diodes 1,3 et 4 .

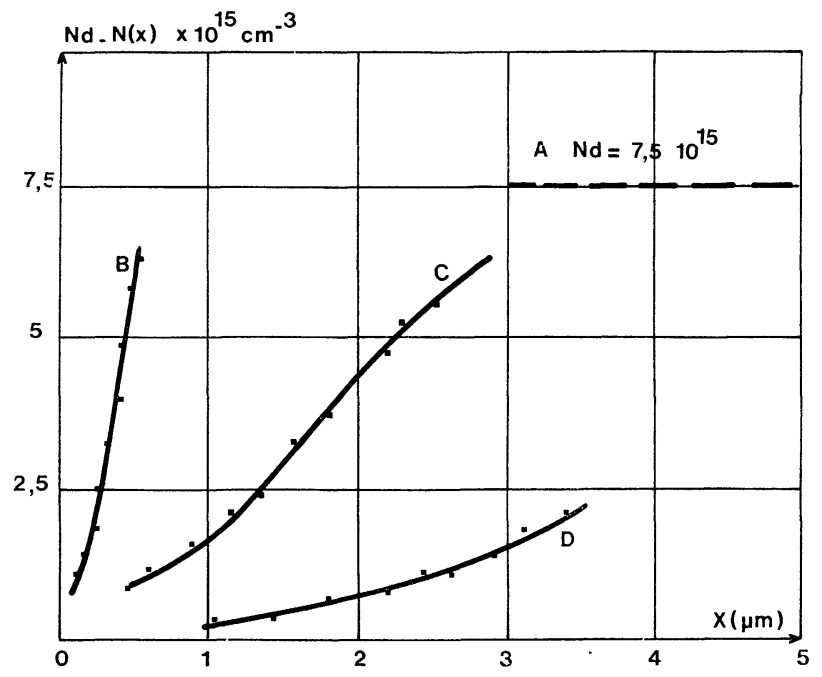

Fig. 7. - Variation de $N \mathrm{D}-N(x)$ en fonction de la distance pour des températures de recuits différentes. A, B, C, D (cf. Fig. 7).

La forme du profil $N_{\mathrm{D}}-N(x)=f(x)$ étant exponentielle, il nous semble logique de rechercher une solution exponentielle plutôt qu'une solution du type erf pour l'équation de Fick. En effet, comme le montre la figure 8 , les courbes $\log N(x)=f\left(x^{2}\right)$

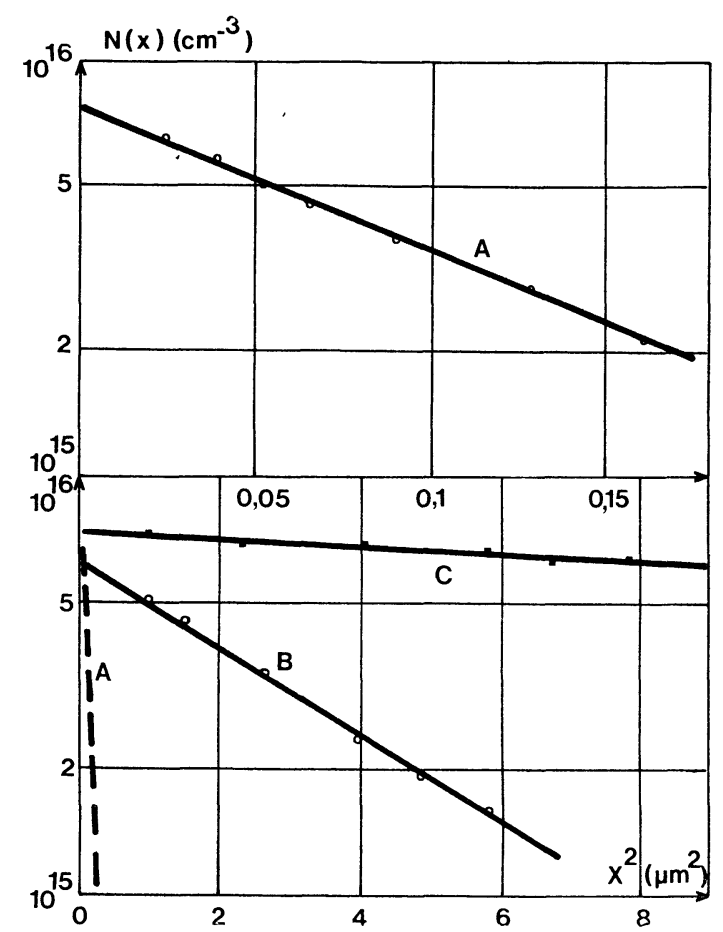

Fig. 8. - Variation de $\log N(x)$ en fonction du carré de la distance pour des températures de recuits différentes. Courbe A $100^{\circ} \mathrm{C} 18 \mathrm{~h} D=5 \times 10^{-15} \mathrm{~cm}^{2} \mathrm{~s}^{-1}$. Courbe B $200^{\circ} \mathrm{C} 10 \mathrm{~h}$ $D=2,6 \times 10^{-13} \mathrm{~cm}^{2} \mathrm{~s}^{-1}$. Courbe C $300^{\circ} \mathrm{C} 6 \mathrm{~h}$ $D=4,5 \times 10^{-13} \mathrm{~cm}^{2} \mathrm{~s}^{-1}$.
( $x$ étant la largeur de la zone de charge d'espace à la tension de polarisation $V$ appliquée) sont des droites.

Le graphe donnant la variation du coefficient de diffusion en fonction de $1 / T$ est donné par la figure 9 . Nous avons calculé une énergie d'activation de $0,75 \mathrm{eV}$ pour les atomes d'argent diffusés.

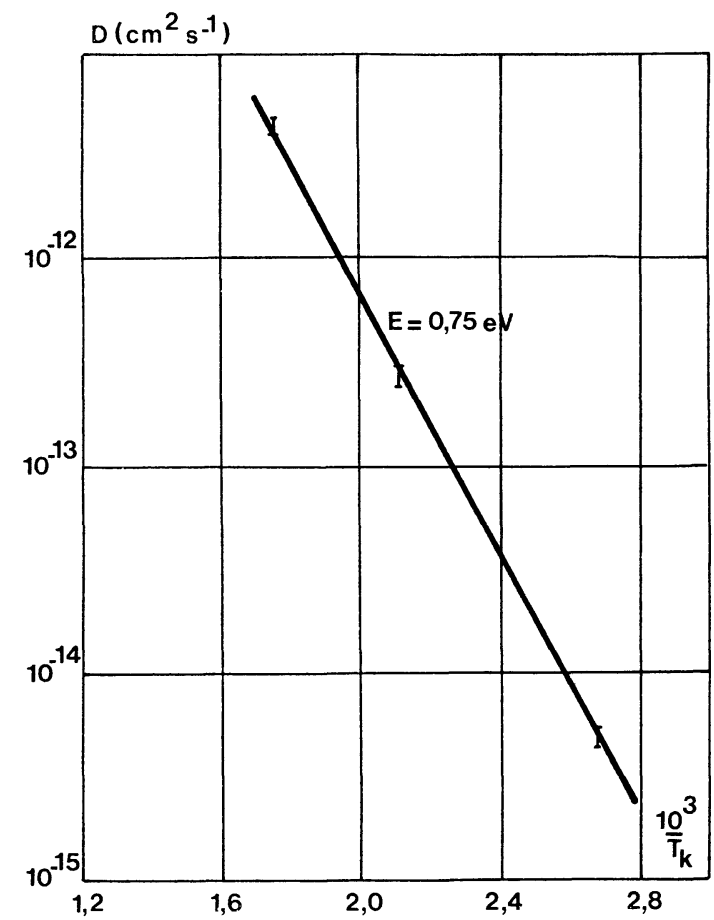

Fig. 9. - Variation du coefficient de diffusion $D$ en fonction de l'inverse de la température. La pente donne une énergie $E=0,75 \mathrm{eV}$.

La figure 10 représente la variation du carré de la largeur de zone de charge d'espace en fonction du temps de diffusion pour une diode à l'argent. Nous observons pendant les premières heures de la diffusion une variation non linéaire.

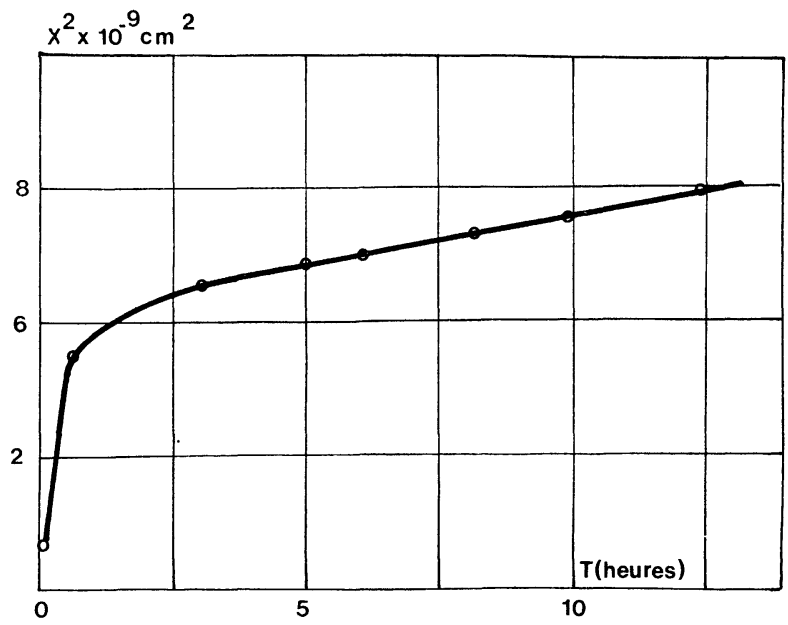

Fig. 10. - Variation du carré de la largeur de la charge d'espace sous une tension de polarisation nulle en fonction du temps de recuit à une température de $200^{\circ} \mathrm{C}$ pour la diode $\mathrm{n}^{\circ} 2 \mathrm{AgCdSIn}$ 
4. Discussion des résultats. - Par sa conception même notre méthode nous permet uniquement de mesurer la variation de la densité de donneurs ionisés. La modification des profils de dopage présentés sur la figure nous amène à penser que les atomes d'argent diffusent en compensant les centres donneurs de densité initialement constante et établissent le profil de donneurs présentés sur cette figure. Nous remarquons d'autre part que l'extrapolation de la courbe donnant la concentration à la surface pour $x=0$ est toujours inférieure ou égale à $N_{\mathrm{D}}$. Il n'y a donc pas inversion du type de conductibilité au voisinage de la surface. En effet, l'existence de CdS de type P obtenue

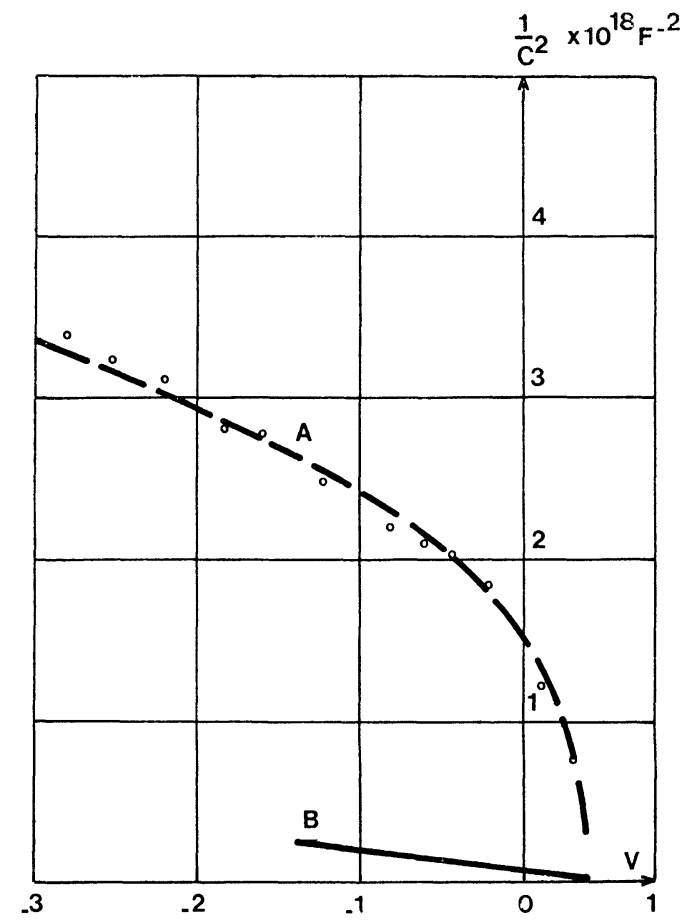

Fig. 11. - Tracé théorique de $1 / C^{2}=f(V)$ en pointillé (courbe A) calculé à partir de l'éq. $(1.16)$; les points expérimentaux pour la diode $\mathrm{AgCdSIn} \mathrm{n}^{\circ} 4$ sont représentés par des cercles. Courbe B diode AgCdSIn avant diffusion. par diffusion d'un élément n'a jamais été observé. D'après Kumar et Kroger [7], les centres donneurs seraient dus surtout aux lacunes de soufre doublement ionisées. Il semblerait donc qu'il y ait compensation de ces lacunes par les atomes d'argent et cela pourrait expliquer la faible valeur du coefficient de diffusion que nous avons mesurée.

Enfin, nous avons introduit dans l'éq. (1.12) tous les paramètres expérimentaux de la diode $n^{0} 4$, c'està-dire le coefficient de diffusion

$$
D=4,5 \times 10^{12} \mathrm{~cm}^{2} \mathrm{~s}^{-1}, \quad V_{\mathrm{D}}=0,44 \mathrm{~V}
$$

et une concentration à la surface extrapolée $N_{\mathrm{S}}=7,5 \times 10^{15} \mathrm{~cm}^{-3}$. La figure 11 montre bien que la variation de $1 / C^{2}=f(V)$ est bien décrite par la précédente équation.

5. Conclusion. - Au cours de ce travail, nous avons étudié des contacts Argent-CdS. Nous avons appliqué les théories de Schottky sur la capacité du contact, métal-semiconducteur.

Nous avons remarqué que le caractère polaire des cristaux de CdS joue un rôle important dans les propriétés électriques du contact métal-semiconducteur. La face cadmium donne des contacts plus proches de ceux décrits par la théorie de Schottky, tandis que la face soufre donne des contacts de mauvaise qualité.

La méthode capacitive nous renseigne parfaitement sur l'évolution des défauts électroniques tels que centres donneurs ou accepteurs dans une région proche de la surface consécutive à l'introduction d'atomes d'argent, mais elle ne nous permet pas pour l'instant de tirer des conclusions concernant les divers sites occupés par les atomes d'argent. Une étude est en cours à ce sujet.

Remerciements. - Les auteurs remercient $M^{\mathrm{me}} F$. Cabane-Boutry et $\mathrm{M}^{\mathrm{r}}$ E. Guyon pour leurs fructueuses remarques et tous les membres du laboratoire pour leur aide incessante.

\section{Bibliographie}

[1] Martinuzzi, S., Cabane-Boutry, F., Sorbier, J.-P. et BRETZNER, J. F., On properties and analysis of CdS$\mathrm{Cu}_{2} \mathrm{~S}$ heterojonctions, Colloque international sur la physique et la chimie des semiconducteurs et des hétérojonctions, Budapest 1970.

[2] Adda, Y., Philibert, J., La diffusion dans les solides (Presses Universitaires de France, Paris) 1966.
[3] HaAs, K. J., Fox, D. C., Katz, J. M., J. Phys. \& Chem. Solids $26(1965) 1779$.

[4] Wolff, G., Tech. Documentary Rapport 11 (1962) 58.

[5] Sullivan, G. A., Phys. Rev. 134 (1969) 796.

[6] BETHE, H. A., Theory of the boundary layer of crystal rectifiers, MIT Radiation Laboratory, Report 43 (1942) 12.

[7] Krumar, V., Kroger, F. A., J. Sol. Stat. Chem. 3 (1971) 387 400 . 